\begin{tabular}{|c|c|c|}
\hline ITC $1 / 47$ & \multicolumn{2}{|c|}{ A Survey on Shadow Detection Techniques in a Single Image } \\
\hline $\begin{array}{l}\text { Journal of Information Technology } \\
\text { and Control }\end{array}$ & Received 2017/05/18 & Accepted after revision 2018/01/12 \\
\hline $\begin{array}{l}\text { pp. } 75-92 \\
\text { DOI 10.5755/j01.itc.47.1.15012 } \\
\text { ๑ Kaunas University of Technology }\end{array}$ & \multicolumn{2}{|c|}{ cross ${ }^{2}$ http://dx.doi.org/10.5755/j01.itc.47.1.15012 } \\
\hline
\end{tabular}

\title{
A Survey on Shadow Detection Techniques in a Single Image
}

\author{
Saritha Murali, Govindan V. K., Saidalavi Kalady \\ Department of Computer Science and Engineering, National Institute of Technology Calicut, Kerala, India. \\ e-mails: saritha.mkv@gmail.com,vkg@nitc.ac.in, said@nitc.ac.in
}

Corresponding author: saritha.mkv@gmail.com

Shadows are inescapable elements in a scene formed due to the presence of an object between the light source and the surface on which it is cast. Appearance of shadows often causes severe issues in computer vision applications like object extraction, and surveillance. Researchers have made effort to device techniques to locate and remove shadows from images and videos. This paper attempts to survey the various shadow detection algorithms for a single image. For the purpose of survey, the notable research work in the literature is classified under five major categories: invariant-based detection, feature-based detection, region-based detection, color model based detection and interactive shadow detection. The survey also includes a qualitative and quantitative evaluation of the methods discussed. As outcomes of the survey, it is observed that, (i) although the works discussed under each of these categories are capable of detecting shadows in different scenarios, the accuracy and time taken need further improvements to make the shadow detection process acceptable for practical applications; (ii) detection of self-shadows and heavily scattered shadows is challenging; (iii) the risk of misclassifying dark objects as shadows should be addressed; (iv) soft shadows that span multiple surfaces pose challenge in accurate detection of shadows.

KEYWORDS: Shadow detection, Invariant image, Feature extraction, Color models, Interactive.

\section{Introduction}

Shadows appear on a surface when light from a source (or multiple sources) is unable to reach the surface due to obstruction by an object. An object may cast shadow on itself (it is then called self-shadow), or on another surface (it is then termed as cast shadow). Although shadows can provide useful cues for estimating scene illumination, finding the geometry of object casting the shadow, locating the light source and so on, their presence may cause hindrance in various image and video processing tasks, such as segmentation, object detection, and video surveillance. Therefore, the removal of shadows is inevitable for the flawless execution of these algorithms. Since shadow removal is aimed at enhancing an image or video to make it suitable for a computer vision task, it is mostly incorporated into the preprocessing stage of these applications. 
The removal of shadows from an image can be considered as a two-stage process, where the detection of shadows is to be performed first, followed by the removal. Most of the works in this area focus on shadows in video [4] and multiple images. Detection and elimination of shadows from a picture is troublesome since the entire cues for detection are to be derived from the single input image. An extensive review of cast shadow detection techniques depending on object/environment and domain was presented by Al-Najdawi et al. [1]. Different moving cast shadow extraction algorithms were studied by Sanin et al. [27]. They classified the algorithms under a feature-based taxonomy and performed a comparative analysis of the techniques. A major attempt in reviewing the shadow detection and removal from real images was done by Sasi and Govindan [29].

\section{Figure 1}

Shadow formation model

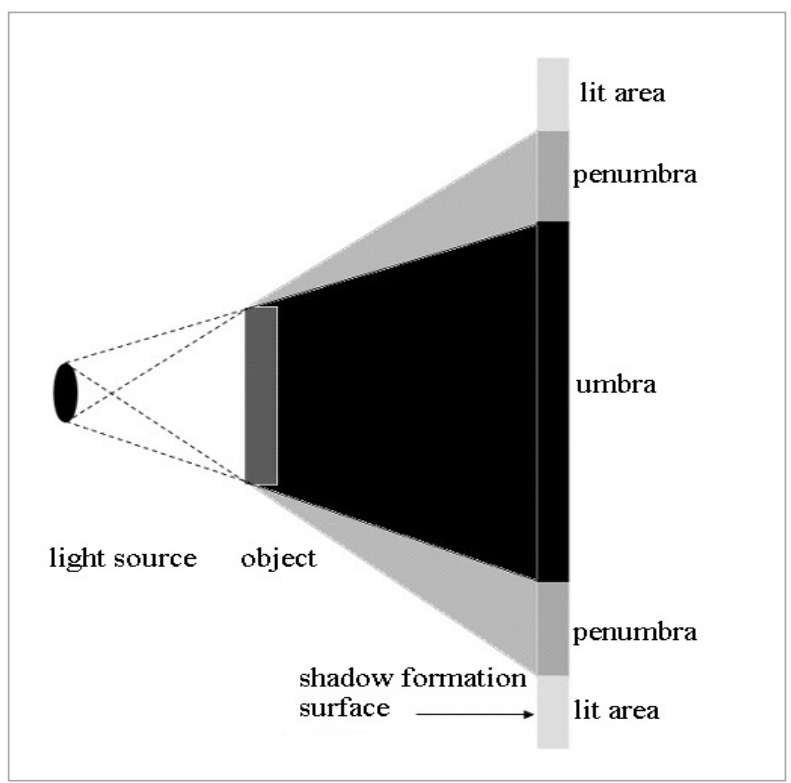

This work attempts to review some of the single image shadow detection techniques available in the literature. In addition to the cast-shadow detection methods [1], our review mentions the algorithms that are capable of detecting self-shadows. In addition, our classification considers both feature-based techniques [27] and non-feature extraction techniques, such as invariant based shadow detection. The rest of the paper is divided into the following sections: first, we present a brief shadow formation model in Section 2. Certain indicative properties of shadows are mentioned in Section 3. This is succeeded by a critical review of different shadow detection algorithms, which are classified into five categories in Section 4. A qualitative and quantitative analysis is done in Section 5. We conclude the survey in Section 6.

\section{The Shadow Formation Model}

Figure 1 illustrates the formation of shadow due to the presence of an object between a non-point light source and the region where the shadow is cast upon. In this scenario, the shadow is seen to be comprised of two regions, the inner umbra and the outer penumbra. Umbra is the dark area in shadow formed by the complete obstruction of light, whereas, penumbra is the lighter area in the shadow formed due to partial blocking of direct light from the source. Since the illumination in the umbra region is much less compared to that in the penumbra and the lit area, it is the darkest shadow region. The texture detail in this region of shadow is mostly corrupted. The penumbra region looks much more diffused, but retains the texture information. The main difficulty in locating the penumbra region arises due to the diffused boundary between the penumbra and the lit area.

An image may be considered to be a composition of two components that represent illumination and reflectance [3]. The illumination component depends upon the light source which illuminates the scene, whereas, the reflectance element is based on the property of the surface or object which is illuminated. Hence, an image $I(x, y)$ could be expressed as

$$
I(x, y)=R(x, y) \cdot L(x, y)
$$

where $R$ and $L$ are the reflectance and the illumination components, respectively. Since shadows are formed due to a lack of illumination, the removal of illumination component will provide a reflectance image which is nearly shadow-free.

\section{The Properties of Shadows}

Understanding the properties of shadows is inevitable in identifying the shadows in an image. Shadow 
regions exhibit various properties that differentiate them from the non-shadow areas in an image. The mean values of various properties for $64 \times 64$ patches of shadow and corresponding non-shadow ground truth of three images from the dataset given in [16] are shown in Table 1.

Table 1

The properties of shadow regions and non-shadow regions

\begin{tabular}{|c|c|c|c|c|c|c|c|}
\hline \multirow[t]{2}{*}{ Property } & \multicolumn{2}{|c|}{$\sum_{\text {Image } 1}$} & \multicolumn{2}{|c|}{ Image 2} & & age 3 & \multirow[t]{2}{*}{$\begin{array}{c}\text { Value in } \\
\text { Shadow area } \\
\text { compared to } \\
\text { Non-shadow }\end{array}$} \\
\hline & $\mathbf{S}^{*}$ & $\mathbf{N S}^{*}$ & $\mathbf{S}$ & NS & $\mathbf{S}$ & NS & \\
\hline RGB Values & {$[95,109,121]$} & {$[159,168,171]$} & {$[64,64,58]$} & {$[143,132,104]$} & {$[73,53,52]$} & {$[160,113,101]$} & Lower \\
\hline Gray Level Intensity & 106 & 166 & 64 & 132 & 60 & 126 & Lower \\
\hline Local Max & 152 & 249 & 101 & 226 & 82 & 201 & Lower \\
\hline Hue & 0.58 & 0.50 & 0.16 & 0.13 & 0.22 & 0.03 & Nearly same \\
\hline Saturation & 0.21 & 0.08 & 0.15 & 0.31 & 0.30 & 0.37 & Lower \\
\hline Brightness & 0.48 & 0.67 & 0.26 & 0.57 & 0.29 & 0.63 & Lower \\
\hline Entropy & 5.44 & 6.51 & 5.76 & 7.10 & 4.23 & 5.32 & Lower \\
\hline Kurtosis & 3.12 & 3.29 & 1.52 & 1.47 & 2.24 & 3.01 & Different \\
\hline Standard Deviation & 10.61 & 22.47 & 20.47 & 54.73 & 6.21 & 16.16 & Lower \\
\hline Skewness & 0.07 & -0.02 & 0.32 & 0.24 & 0.37 & 0.88 & Different \\
\hline Graylevel Variance & 112 & 504 & 419 & 2995 & 38 & 261 & Lower \\
\hline
\end{tabular}

${ }^{*}$ S- shadow NS-Non-shadow

It is observed that shadow regions have lower values for RGB, graylevel intensity, standard deviation, variance, local maximum, and brightness since these values depend on the illumination and the shadow regions are less illuminated than the surroundings. The hue value that indicates the dominant color of a surface remains nearly the same in both shadow and shadowless regions [18]. Difference in skewness values arises due to difference in the asymmetries in shadow and non-shadow regions [41]. Since shadow areas are darker, their entropy [41] and saturation values are less. Many of these properties are explored by the researchers to separate the shadow regions from the shadowless regions in an image.

\section{Shadow Detection Methods}

The initial stage in the elimination of shadows from an image involves correctly locating the shadow ar- eas. Numerous works are available in the literature for detection of shadows in videos, aerial images, and outdoor images. In this paper, various techniques used for detecting the shadows in an image are discussed.

We have identified five classes of detection algorithms:

A. Invariant-based shadow detection;

B. Feature-based shadow detection

i. feature extraction based

ii. feature learning based;

C. Region-based shadow detection;

D. Color model based shadow detection;

E. Interactive shadow detection.

Some of the noted works in literature are classified under these categories and explained further in this section. 


\section{A. Invariant-Based Shadow Detection}

Most of the early works in shadow detection from a single image focused on finding an invariant image representation in which shadows are absent or less visible. The theory of invariant image is based on the concept that shadows occur in lesser illuminated areas in an image. Hence, generating an illumination-invariant image could provide useful information on the shadow areas. Since shadows are absent in the invariant image, edge-map of illumination invariant image will have only object edges. The fundamental issue is to find a mechanism to generate such a representation.

The most prominent work in this category was done by Finlayson et al. [9]. They deployed a 1-Dimensional grayscale illumination-invariant representation [8] of an image that is obtained by projecting the 2-Dimensional chromaticity along a line upright the direction of illumination change. Edge detection was done on both illumination-invariant and original images. The differences in edge-maps of the images resulted in shadow edges. The problem with this method was in determining the direction of illumination change which required calibrated cameras. Later, Finlayson et al. [7] demonstrated that the correct invariant direction is the one which will result in least entropy in the 1-Dimensional greyscale invariant image. The 1-Dimensional invariant representation did not carry color information. Hence, a method to generate a 2-Dimensional invariant image that retains certain color information was put forward in [10]. He and Chu [17] used Fisher Linear Discriminant (FLD) to find the invariant direction. Even though the detection results are not that acceptable, the authors verified that FLD can be used to generate invariant image. Two adjacent pixels on a surface form an illuminant discontinuity pair if they appear with dissimilar intensities in original image, but are aligned on same direction in the log chromaticity space. Lu and Drew [21] formulated an illuminant discontinuity measure,

$$
k_{i j}=\left|\frac{<e_{i j}, e_{0}>}{\left\|e_{i j}\right\|\left\|e_{0}\right\|}\right|,
$$

where $e_{i j}$ is a vector that associates the log ratios of adjacent pixels $i, j$; and $e_{0}$ is the illuminant direction.
This measure is obtained from intensity and chromaticity cues, and is used to label shadow pixels by Markov Random Field (MRF) with graph-cut optimization. Tian et al. [34] formulated a Tricolor Attenuation Model (TAM) considering the Spectral Power Distribution (SPD) of light during daytime and light from the sky to detect shadows in outdoor images. They developed an approximate shadow invariant by means of an RGB to greyscale transformation;

$Y=\log \left(\frac{\max \left[\begin{array}{lll}F_{R} & F_{G} & F_{B}\end{array}\right]}{\min \left[\begin{array}{lll}F_{R} & F_{G} & F_{B}\end{array}\right]+1}\right)$,

where $\left[F_{R} F_{G} F_{B}\right]$ indicates the tricolor value of a pixel in an RGB image, and $\mathrm{Y}$ is the resultant grayscale image. This method works effectively on complex outdoor scenes also.

\section{Discussion}

Invariant-based shadow detection techniques aim to find a representation that is free from shadows. These algorithms require prior knowledge of illuminant direction or calculation of illuminant direction by camera calibration [9], entropy minimization [7], or Fisher Linear discriminant [17]. Most of these algorithms work under the assumption of Planckian light, Lambertian surfaces and linear illumination. The generation of invariant images demands high quality input images that are noiseless and uncompressed. Detection results are also affected by the edge detection algorithms used to create the edgemap of the original image and its invariant. However, these methods generally incur less time for generating the results. A summary of the features of the algorithms mentioned in this category is included in Table 2.

\section{B. Feature-Based Shadow Detection}

Shadow regions possess several features that differentiate them from the non-shadow regions. These include features measured from color, edge, photometric properties, geometry, texture, shadow-variant properties, and shadow invariant properties. Certain methods use feature extraction to identify shadow areas, whereas others use feature learning to train a classifier to locate the shadow areas in an image. The algorithms in this category are hence grouped into 
Table 2

A summary of invariant based shadow detection methods

\begin{tabular}{l|l|l}
\hline \multicolumn{1}{c|}{ Author } & \multicolumn{1}{c|}{ Invariant Calculation } & \multicolumn{1}{c}{ Key techniques in detection } \\
\hline Finlayson et al. [9] & $\begin{array}{l}\text { Projecting data along invariant } \\
\text { direction chosen by camera } \\
\text { calibration }\end{array}$ & $\begin{array}{l}\text { 1D illumination invariant, SUSAN edge detector } \\
\text { Detection: difference in edge maps of original and } \\
\text { invariant images }\end{array}$ \\
\hline Finlayson et al. [10] & $\begin{array}{l}\text { 2D invariant derived from 1D } \\
\text { invariant [9] }\end{array}$ & $\begin{array}{l}\text { 2D illumination invariant, 3D invariant } \\
\text { Detection: difference in edge maps of original and } \\
\text { invariant images }\end{array}$ \\
\hline Lu and Drew [21] & $\begin{array}{l}\text { Illuminant direction by } \\
\text { calibration or entropy } \\
\text { minimization }\end{array}$ & $\begin{array}{l}\text { Mean Shift filtering, graph cut } \\
\text { Detection: illuminant discontinuity measure, MRF } \\
\text { segmentation }\end{array}$ \\
\hline Tian et al. [34] & $\begin{array}{l}\text { Using tricolor vector of pixel } \\
\text { values }\end{array}$ & $\begin{array}{l}\text { Invariant image,watershed segmentation, Tricolor } \\
\text { Attenuation Model } \\
\text { Detection: Thresholding on TAM }\end{array}$ \\
\hline He and Chu [17] & $\begin{array}{l}\text { Projecting data along invariant } \\
\text { direction by FLD }\end{array}$ & $\begin{array}{l}\text { K-means clustering, Fisher Linear Discriminant, Canny } \\
\text { edge detector } \\
\text { Detection: difference in edge maps of original and } \\
\text { invariant images }\end{array}$ \\
\hline
\end{tabular}

two subsections, namely feature extraction based detection and feature learning based detection.

\section{i. Feature Extraction Based Shadow Detection}

By using a single feature it is often difficult to identify whether a pixel or region belongs to shadow or non-shadow region. Therefore, most of the feature extraction based algorithms intent to determine the best combination of features that can correctly detect the shadow regions in an image.

Salvador et al. [26] modelled shadow detection as a multi-stage process using color invariant and geometric features. The algorithm begins by testing a hypothesis formulated over the concept that shadows blacken the area on which they are formed. This is followed by a verification of the result using color invariance and shadow location with reference to the object. The color invariant used in their technique was c1c2c3 model [11]. The method often fails to determine the shadow lines for soft shadows. A method to locate shadows using a combination of colour and edge features was put forward by Golchin et al. [13]. Shadow pixels were detected in HSI, modified c1c2c3, YCbCr color spaces, and hue difference of the background and the foreground regions separately. Followed by this, detection was done based on edge information using Sobel operator. Finally, all the detection results were combined using Boolean AND. Even though the method requires the background image as extra input, it is found to outperform the detection using color or edge feature alone.

Detection of shadows by using bright channel is another work in this category. The concept of a bright channel lies on the assumption that any image segment will have pixels with values close to the incoming radiance in at least one color channel. Panagopoulos et al. [24] used this property to extract shadows from an image. The bright channel formed is refined by computing confidence of shadow areas using shadow features such as hue or by using Markov Random Field (MRF) model. The refined bright channel is then threshold into a binary image to get the shadow regions.

Tian et al. [33] formulated four physical characteristics of shadows found in outdoor scenes based on RGB color matching and SPD of illumination. Based on these features, three shadow verification criteria were derived to detect shadows in a single image. The authors claim that spectrum ratios provide good physical cues on shadows since they do not depend on wavelength and reflectance, however, it fails for 
Table 3

A summary of feature extraction based shadow detection methods

\begin{tabular}{l|l|l}
\hline \multicolumn{1}{c|}{ Author } & \multicolumn{1}{c}{ Features } & \multicolumn{1}{c}{ Key techniques in detection } \\
\hline Salvador et al. [26] & $\begin{array}{l}\text { Spectral (color invariance) and } \\
\text { geometric features }\end{array}$ & $\begin{array}{l}\text { Sobel operator, photometric color invariant features, spatial } \\
\text { constraints. } \\
\text { Detection: Thresholding }\end{array}$ \\
\hline Panagopoulos et al. [24] & $\begin{array}{l}\text { Bright channel cue, } \\
\text { illumination invariant features: } \\
\text { Hue, normalized RGB, c1c2c3 }\end{array}$ & $\begin{array}{l}\text { Bright channel, Markov Random Field } \\
\text { Detection: Thresholding }\end{array}$ \\
\hline Golchin et al. [13] & $\begin{array}{l}\text { Color Features: HSI, modified } \\
\text { c1c2c3,YCbCr, hue difference } \\
\text { Edge Features }\end{array}$ & $\begin{array}{l}\text { Sobel operator, Morphological open operator } \\
\text { Detection: Thresholding }\end{array}$ \\
\hline Dong et al. [5] & $\begin{array}{l}\text { Soft shadow features: center } \\
\text { position, orientation and width } \\
\text { of penumbra }\end{array}$ & $\begin{array}{l}\text { Watershed segmentation, Canny edge detector, level-set } \\
\text { optimization } \\
\text { Detection: intensity fitting }\end{array}$ \\
\hline Tian et al. [33] & Spectrum ratio properties & $\begin{array}{l}\text { Canny edge detector } \\
\text { Detection: Thresholding and four new shadow properties }\end{array}$ \\
\hline
\end{tabular}

over-exposed or noisy shadow regions. Dong et al. [5] attempted to model soft shadows using three features - center, orientation and width of penumbra. These measures were derived by fitting the intensity changes to a sigmoid function, and were used to detect shadows. The major pitfall of this method is the difficulty in detecting shadows on multiple surfaces and uneven surfaces.

\section{ii. Feature Learning Based Shadow Detection}

The feature based techniques that use a trained classifier to separate the shadow and shadowless regions are discussed in this section. Some of these works use a predefined set of features for training while others automatically learn the most relevant features for classification.

Gijsenij and Gevers [12] experimentally proved that a unification of geometric and photometric features could enhance the detection results compared to using either of the features alone. They used a nearest neighbour classifier to identify shadow patches in an image based on photometric and geometric features. The results show that the best geometric features that aid in detection are diagonal edges or absence of edges in the patch.

A method to detect shadows cast on ground was proposed by Lalonde et al. [20]. Initially, a decision tree classifier is trained on the basis of various color and intensity features computed around the image edges. A 48-dimensional feature vector is generated for each pixel. Conditional Random Field (CRF) incorporated with a scene layout descriptor is then used to group the detected shadow edges. The method often fails for shadows of thin objects.

Zhu et al. [41] formulated a feature learning method to locate shadows in a monochromatic image. They used such shadow variant features as intensity difference, local max, smoothness and skewness; such shadow-invariant features as gradient similarity and texture similarity; and such near black features as discrete entropy and edge response to train the classifier. Each pixel is labelled as shadow or not based on classifier built by integrating Boosted Decision Tree (BDT) with binary CRF (BCRF). The authors conclude that the integrated classifier outperforms BDT or BCRF alone.

A low-cost shadow detection technique that can be incorporated into vehicle vision system for clear path detection was developed by $\mathrm{Wu}$ et al. [37]. They generated illumination invariant, illumination direction and neighbouring similarity features of the edge patch candidates in an image and classified the patches as shadow or non-shadow using a trained SVM classifier. Further, spatial patch smoothing was used to refine the detection result. Khan et al. [19] used a deep learning technique for automatic learning of region- 
al and across-boundary features using two different ConvNets (Convolutional deep Neural Networks). The unary and pairwise potentials of these features are combined in a CRF framework and shadow labelling is done using Maximum a Posteriori (MAP) estimate. A method to learn the most important structure features of shadow boundaries using a structured Convolutional Neural Network (CNN) framework was given by Shen et al. [31]. The authors introduced shadow and bright values to approximately construct the complicated relation among the image regions. Shadow detection is formulated as a least square optimization of non-local region constraints.

\section{Discussion}

The feature-based techniques for shadow detection provide best results when the optimal set of features is correctly identified. Determining this optimal set is a difficult task since increasing the feature count to improve the detection accuracy contributes to a rise in computational load on the detection system, whereas, reducing the number of features will degrade the detection results itself. In addition, the features often extract complex properties of image regions. Even though the feature learning algorithms are seen to give more accurate results compared to the invariant based or other non-learning approaches, the time and memory requirements for training the classifier are usually very high. Moreover, the performance of trained classifiers relies on size and quality of training dataset. The results given in [20] illustrate a case in which the shadow regions are undetected due to the non-inclusion of varying colored surfaces in the training set. A summary of the features extracted and major techniques used in shadow identification for feature based methods are given in Table 3 and Table 4.

Table 4

A summary of feature learning based shadow detection methods

\begin{tabular}{|c|c|c|}
\hline Author & Features & Key techniques in detection \\
\hline Gijsenij et al. [12] & $\begin{array}{l}\text { Photometric: Quasi-invariants, Physics-based } \\
\text { invariants, Color constancy at pixel, Normalized- } \\
\text { RGB. } \\
\text { Geometric: SIFT,LBP,GLCM }\end{array}$ & $\begin{array}{l}\text { Maximum posterior probability } \\
\text { Classifier: Nearest-neighbour }\end{array}$ \\
\hline Lalonde et al. [20] & $\begin{array}{l}\text { Boundary features: brightness ratios, color } \\
\text { intensity ratios, texture, skewness, edge } \\
\text { sharpness, scene layout cues }\end{array}$ & $\begin{array}{l}\text { Bilateral filter, watershed segmentation, Canny } \\
\text { edge detector, CRF optimization } \\
\text { Classifier: logistic regression of Adaboost } \\
\text { decision tree }\end{array}$ \\
\hline Zhu et al. [41] & $\begin{array}{l}\text { Shadow-variant: intensity difference, local max, } \\
\text { smoothness, skewness } \\
\text { Shadow-invariant: gradient, texture similarity } \\
\text { near black features: discrete entropy, edge response }\end{array}$ & $\begin{array}{l}\text { MRF labeling } \\
\text { Classifier: Boosted decision tree with binary CRF }\end{array}$ \\
\hline Wu et al. [37] & $\begin{array}{l}\text { Illumination invariant: reflectance, color } \\
\text { variance, gradient entropy } \\
\text { Illumination direction: } 2 \mathrm{D} \text { log chromaticity } \\
\text { Neighbouring similarity: gradient, texton }\end{array}$ & $\begin{array}{l}\text { Adaptive gradient threshold to get edge } \\
\text { candidates, Spatial patch smoothing } \\
\text { Classifier: SVM }\end{array}$ \\
\hline Khan et al. [19] & Regional and across-boundary features & $\begin{array}{l}\text { SLIC superpixel extraction, gPb boundary } \\
\text { detector, MAP estimate, CRF labeling } \\
\text { Classifier: } 2 \text { CNNs to detect shadow regions and } \\
\text { edges }\end{array}$ \\
\hline Shen et al. [31] & Local structure information of shadow edge & $\begin{array}{l}\text { Canny edge detector, shadow/bright measure, } \\
\text { Quick Shift segmentation } \\
\text { Classifier: structured CNN }\end{array}$ \\
\hline
\end{tabular}




\section{Region-Based Shadow Detection}

Traditional methods for shadow detection considered it to be a pixel-labeling or edge-classification problem. Later, the region-based techniques evolved in which the segmented regions in an image were labeled as shadows or non-shadows based on the properties of individual regions as well as similarity with other regions. The single region classification considers features like color, texture, and brightness, whereas pairwise classification is done by comparing the properties of two regions such as histograms of color and texture, intensity ratios, chromatic alignment, and distance between them. The major works in region-based detection are examined in this section.

Guo et al. [16] modeled a region based technique to detect shadows from natural scenes. They trained a single region classifier using SVM with $\chi 2$ kernel and a pairwise classifier using SVM with RBF kernel. An energy function that integrates the single region classifier and pairwise classifier predictions was then minimized by using graph cut. Their pairwise classifiers compare regions of same material with same illumination and different illumination. The key advantage of this method is that the pairwise classifier considers non-adjacent regions also. Yuan et al. [40] used a physical model called color shade descriptor [30] in L*a*b* color space to find pairwise potentials of shadow regions. The descriptor is based on scene reflectance. Logistic regression of Adaboost with 16-node decision trees is trained for single region classification. These singleton and pairwise potentials are incorporated into a Conditional Random Field (CRF) model. The method performs poorly when the surface is uneven or is deficient in color information.

An SVM classifier with multi-kernel model was used to learn individual shadow regions in the work by Vicente et al. [35]. Their multi-kernel model is an integration of the separate kernels for each feature. The pairwise classifiers used in this method compare only the adjacent regions of same material with same and different illumination. A boundary classifier was also introduced to deal with the shadow boundaries on a surface. All these classifiers are further optimized in an MRF framework. Similar to [16], this approach also gives same weight to all features computed. A region-based shadow detection technique that learns the feature weights along with the classifier was proposed by Vicente et al. [36]. They trained a Least Square Support Vector Machine (LS-SVM) to find the probability of each region to be a shadow region, and contextual relation between neighboring regions to find the pairwise potential. The labeling was optimized by minimizing the leave-one-out error. The classical split-and-merge algorithm with fuzzy predicate was used to detect shadow regions in Sasi and Govindan [28]. The method splits and merges adjacent homogeneous quadtree blocks in the image based on the predicate. Entropy, edge response, standard deviation, and mean of quadtree blocks were considered for building the fuzzy predicate.

\section{Discussion}

The key idea behind region-based shadow detection techniques is that the correctness of detection can be improved by incorporating a pairwise classifier that classifies a region into shadow or non-shadow by comparing it with the other regions in the image. Techniques that compare adjacent regions only and those that consider non-adjacent regions were mentioned in this section. Most of these algorithms work on the basis of learned region features. Hence, the computational load is generally high for this category of algorithms. Table 5 consolidates the single region features and pairwise features considered for these methods and the classifiers used for detection.

\section{Color-Model Based Shadow Detection}

Transforming an image from one color model to another may provide vital cues for detecting shadows in it. The color model based shadow detection techniques initially convert an RGB image to another color model followed by detection in the new model. Normalized RGB, c1c2c3 and HSV are the most commonly used color models for shadow detection since they produce image invariant to shadows. Other color spaces used are CIEL*u*v* and CIEL*a*b* due to their similarity with human perception.

One of the earliest works in this category was done by Salvador et al. [25]. In their method, object contours are generated using edge-map of the c1c2c3 color invariant of the actual image. The edge-map of a luminance image is used to extract dark regions that are shadow candidates since the luminance image is af- 
Table 5

A summary of region based shadow detection methods

\begin{tabular}{l|l|}
\multicolumn{1}{c|}{ Author } & \multicolumn{1}{c|}{ Classifier } \\
Guo et al. [16] & $\begin{array}{l}\text { Single region: SVM with } \chi^{2} \text { kernel; } \\
\text { Pairwise: SVM with RBF kernel }\end{array}$ \\
\hline Vicente et al. [35] & $\begin{array}{l}\text { Single-region: multikernel SVM; } \\
\text { Boundary, pairwise classifiers: } \\
\text { SVM (RBFkernel) }\end{array}$ \\
\hline Yuan et al. [40] & $\begin{array}{l}\text { Single-region: logistic regression, } \\
\text { decision trees; } \\
\text { Pairwise: color shade descriptor }\end{array}$ \\
\hline Vicente et al. [36] & $\begin{array}{l}\text { Single-region: Least Square SVM } \\
\text { Pairwise: Contextual cues }\end{array}$ \\
\hline $\begin{array}{l}\text { Sasi and } \\
\text { Govindan [28] }\end{array}$ & $\begin{array}{l}\text { Fuzzy Split and Merge using Adaptive } \\
\text { Neuro-Fuzzy Inference System }\end{array}$ \\
\hline
\end{tabular}

fected by shadows. The dark regions in both the cases are then classified into self-shadow or cast shadow based on their presence in object contours. This method works for shadows cast on a flat, non-textured surface only, and the object casting the shadow is expected to be within the image. Figov et al. [6] used a multi-resolution approach in which Fuzzy $C$-means clustering followed by shadow segmentation is done on a lower resolution CIEL* $u^{*} v^{*}$ color representation of the image. This segmentation is then incorporated to the original image to identify the shadow areas. They used a simple Euclidean distance measure to identify regions with similar chrominance and varying luminance given in Equation (4):

$$
||(L *, u *, v *) \|=\sqrt{(L *)^{2}+(u *)^{2}+(v *)^{2}} .
$$

Since a reduced size image is used, the algorithm demands that the original image should not be over-compressed.

An attempt to identify vague and hard shadows from an image was done by $\mathrm{Xu}$ et al. [39]. For identifying hard shadows, normalized RGB (L2 norm) and a 1-Dimensional invariant image generated by using [10] were used. The vague shadows were detected by thresholding the image gradient. The normalized RGB was computed as follows:
Features

Single region: Color and texture histograms

Pairwise: Color and texture histogram distances, intensity ratio, chromatic alignment, distance

Single region: Color and texture histograms

Pairwise: distances between color and texture histograms

Single region: Color, brightness and texture histograms Pairwise: Local maxima of color distribution

Single region: Color, intensity, texture histograms

Pairwise: Color and texture histogram distances, RGB ratios

Entropy, edge response, standard deviation, mean of quadtree

$$
\begin{aligned}
& r^{\prime}=\frac{r}{\sqrt{r^{2}+g^{2}+b^{2}}}, \\
& g^{\prime}=\frac{g}{\sqrt{r^{2}+g^{2}+b^{2}}}, \\
& b^{\prime}=\frac{b}{\sqrt{r^{2}+g^{2}+b^{2}}} .
\end{aligned}
$$

The detected shadow masks are combined to form reintegrated shadow mask.

Murali and Govindan [22] detected shadows in CIE $L^{*} \mathrm{a}^{*} \mathrm{~b}^{*}$ color space based on luminance value and observed that the B-channel values (yellow to blue ratio) are lesser in shadow regions compared to the non-shadow regions. This method gives spurious labeling since it classifies each pixel as shadow and non-shadow without considering the neighboring pixels.

\section{Discussion}

The primary focus of color model based detection techniques is to identify cues that can aid in detecting shadows in a color space different from that of the original image. Color models L*a* $b^{*}$ and $L^{*} u^{*} v^{*}$ have separate luminance component that makes them useful for this purpose. While the algorithms that use col- 


\section{Table 6}

A summary of color model based shadow detection methods

\begin{tabular}{|c|c|c|}
\hline Author & Color model & Key techniques in detection \\
\hline Figov et al. [6] & CIE L* $u^{*} v^{*}$ & $\begin{array}{l}\text { Segment features: area, border length, intensity with respect } \\
\text { to neighbor segments, color ratio, brightness ratio } \\
\text { - Fuzzy c-means clustering }\end{array}$ \\
\hline Salvador et al. [25] & c1c2c3 color invariant & Luminance and color information \\
\hline Xu et al. [39] & $\begin{array}{l}\text { normalized RGB } \\
\text { (L2 norm) }\end{array}$ & $\begin{array}{l}\text { Vague shadows: gradient thresholding; } \\
\text { Hard shadows: color invariant thresholding } \\
\text { - 1D invariant image, Gaussian smooth filter }\end{array}$ \\
\hline Murali and Govindan [22] & CIE L*a*b* & Thresholding on $\mathrm{L}$ and $\mathrm{B}$ channel values \\
\hline
\end{tabular}

or models are simple and fast, most of them misclassify dark regions in the image as shadows. The major features are summarised in Table 6.

\section{E. Interactive Shadow Detection}

Fully automatic shadow detection is yet a challenging problem primarily due to the difficulty in determining whether an area in an image is dark by itself or due to shadow cast on it. Interactive shadow detection techniques let the users incorporate their knowledge in the detection task. This section gives a brief review of five shadow detection techniques that need user input.

Wu and Tang [38] proposed a shadow extraction technique based on quadmap input by the user. The quadmap requires the user to mark four different regions in an image: shadow, non-shadow, uncertain and excluded regions. This input is used in a Bayesian framework to extract the shadow. A method to find shadows in outdoor image with a reduced user input was given by Nielsen and Madsen [23]. In this ap- proach, the user should mark a shadow surface and its sunlit counterpart which is used to compute an alpha parameter. The shadow region is modeled as a function of sunlit region using an alpha overlay and the edges were detected by thresholding based on intensity and illuminant direction. The method often fails for high frequency texture surface.

In the shadow detection technique by Arbel and HelOr [2], region growing using SVM is done separately on the multiple shadow and non-shadow coordinates input by the user. These detected shadow regions are later combined to form the shadow mask. The detection becomes difficult for highly broken shadows, and those on multiple surfaces. One of the simplest interactive methods was proposed by Shor and Lischinski [32] in which the user had to indicate the shadow region by a bare mouse click. The shadow mask is derived from the clicked area by iterative region growing and an illumination invariant distance measure. This technique works only for a surface which has

Table 7

Summary of interactive shadow detection methods

\begin{tabular}{l|l|l}
\multicolumn{1}{c|}{ Author } & \multicolumn{1}{c}{ User-input } & \multicolumn{1}{c}{ Key techniques in detection } \\
\hline Wu and Tang [38] & Quadmap & Bayesian method \\
\hline Nielsen and Madsen [23] & Shadow and sunlit counterpart & $\begin{array}{l}\text { Graph cut energy minimization, alpha overlay } \\
\text { shadow model, thresholding }\end{array}$ \\
\hline Shor and Lischinski [32] & A click in shadow area & $\begin{array}{l}\text { Region growing, illumination invariant } \\
\text { distance measure }\end{array}$ \\
\hline Arbel and Hel-Or [2] & $\begin{array}{l}\text { Mark pixels in shadow and non- } \\
\text { shadow areas }\end{array}$ & SVM learning, region growing \\
\hline Gong et al. [15] & A stroke on umbra segment & $\begin{array}{l}\text { Illumination image, sparse-field active } \\
\text { contour, region growing }\end{array}$ \\
\hline
\end{tabular}


both shadowed and non-shadowed areas.

A recent work by Gong et al. [15] requires the user to highlight a portion of umbra by one rough stroke. The segment was then used to detect shadow boundaries by region growing on an illumination image constructed from HSV, LUV, and $\mathrm{YCbCr}$ color spaces. The method is found to work well for non-uniform umbra illumination.

\section{Discussion}

In general, the interactive shadow detection techniques are simpler than automatic detection since an initial indication of shadow region is provided by the user. However, these methods become annoying for the user if the image has multiple disjoint shadow regions. In this case, the user should give hint on each disjoint shadow region. Since the user himself/ herself provides clue on the shadow regions, there is less chance of misclassifying dark objects that are non-adjacent to shadow regions. The techniques discussed are outlined in Table $\%$.

\section{Comparison}

This section begins with a concise explanation of the most widely used shadow detection datasets. This is succeeded by a comparison of the various shadow detection techniques discussed above. The comparison is done both qualitatively and quantitatively.

\section{Shadow Detection Datasets}

The major datasets available for shadow detection and removal in single images are listed below:

1 UCF dataset: 355 images and corresponding manually-labeled ground truths given by [41].

2 UIUC dataset: 108 natural scenes taken under different illumination and their ground truth by [16].

3 CMU dataset: 135 outdoor images with shadow boundary annotations given by [20].

4 Dataset by [14]: 214 images with ground truth.

\section{Qualitative Analysis}

Some of the works in literature consider shadow detection as a pixel-labeling problem, while others perform detection at patch-level, region-level or edge level. A qualitative summary of the algorithms discussed under each category is given in Table 8. Furthermore, successful and failure cases in shadow detection under different scenarios are demonstrated in Table 9 and Table 10. Table 9(a) gives a comparison of the detection results of shadow region-growing technique and pixel-based detection. It is observed that the pixel-labeling technique used by [22] gives spurious result since the method considers properties of individual pixels without considering the neighboring pixels during classification.

Many of the techniques impose constraints on the image quality, lighting conditions, and surface properties. Some of the detection techniques need the input image to be segmented using standard algorithms. Watershed segmentation was used in [34, 5] and [20]. In these cases, the quality of shadow detection depends on the segmentation algorithm adopted. In addition, edge detectors such as Canny [10, 17, 33, 5, 31], SUSAN [9, 20], and Sobel [26, 13] were used for initial edge detection in a few works. The inaccuracies in the edge detection also contribute to spurious edges in shadow detection results. Table 9(b)(iii) illustrates a case where Canny edge detector was used to extract edges. Another example for spurious edges is given in Table 9(c)(iii). Dependence on the surface properties is another criterion that aids in comparison of shadow detection algorithms. A situation where detection fails due to unusual surface color is shown in $\mathrm{Ta}-$ ble 9(d)(iii). This happened since Lalonde et al. [20] used a learning-based technique to locate shadows present in outdoor images where the training set was built with images in which materials constituting the surface are expected to be made of selected materials only. Surface geometry also contributes to efficient shadow detection [40].

An example of dark region misclassification is given in Table 9(e)(iii). The input image has an object which has a dark area similar to the color of shadows in the image. This has led to the misclassification by Guo et al. [16]. Conversely, the feature learning method proposed by Shen et al. [31] was able to classify the non-shadow regions correctly. Dark region misclassification for large dark areas occurs in the method by Panagopoulos et al. [24].

Self-shadow detection is also not addressed by some of the works. A failure case is demonstrated in Table 9(f) (iii) and its correct detection is given in Table 9(f)(ii).

Soft shadows that span multiple surfaces also pose 


\section{Table 8}

A qualitative summary of shadow detection methods

\begin{tabular}{|c|c|c|c|c|c|c|c|c|c|c|}
\hline Invariant based & level & SD & SS & HS & DS & DM & LD & SR & ER & $\mathbf{L}$ \\
\hline Finlayson et al. [9] & pixel & & x & $\checkmark$ & $\checkmark$ & & $\checkmark$ & $x$ & $\checkmark$ & x \\
\hline Finlayson et al. [10] & pixel & & $x$ & $\checkmark$ & $\checkmark$ & & $\checkmark$ & $x$ & $\checkmark$ & $x$ \\
\hline Lu and Drew [21] & pixel & & x & $\checkmark$ & $\checkmark$ & & $\checkmark$ & $x$ & $x$ & $x$ \\
\hline Tian et al. [34] & region & $\checkmark$ & $\checkmark$ & $\checkmark$ & $x$ & & $\checkmark$ & x & $x$ & $x$ \\
\hline He and Chu [17] & pixel & & $\checkmark$ & $\checkmark$ & $x$ & $x$ & $\checkmark$ & $\checkmark$ & $\checkmark$ & $x$ \\
\hline Feature Extraction based & level & SD & SS & HS & DS & DM & LD & SR & ER & $\mathbf{L}$ \\
\hline Salvador et al. [26] & pixel & x & $\checkmark$ & $\checkmark$ & & $x$ & $x$ & $x$ & $\checkmark$ & $x$ \\
\hline Panagopoulos et al. [24] & patch & & & $\checkmark$ & $\checkmark$ & M & $\checkmark$ & $x$ & $x$ & $x$ \\
\hline Golchin et al. [13] & pixel & $\checkmark$ & $\checkmark$ & $\checkmark$ & & & $x$ & $x$ & $\checkmark$ & X \\
\hline Dong et al. [5] & edge & & $\checkmark$ & $\checkmark$ & $\checkmark$ & $x$ & $\checkmark$ & $\checkmark$ & $\checkmark$ & $x$ \\
\hline Tian et al. [33] & edge & x & $x$ & $\checkmark$ & $\checkmark$ & $x$ & $\checkmark$ & $x$ & $\checkmark$ & $x$ \\
\hline Feature learning based & level & SD & SS & HS & DS & DM & LD & SR & ER & $\mathbf{L}$ \\
\hline Gijsenij et al. [12] & patch & & & $\checkmark$ & & & $\checkmark$ & & $\checkmark$ & $\checkmark$ \\
\hline Lalonde et al. [20] & edge & x & $\checkmark$ & $\checkmark$ & $\checkmark$ & & $\checkmark$ & $\checkmark$ & $\checkmark$ & $\checkmark$ \\
\hline Zhu et al. [41] & pixel & & $\checkmark$ & $\checkmark$ & $x$ & $x$ & $x$ & & $x$ & $\checkmark$ \\
\hline Wu et al. [37] & patch & X & $\mathrm{M}$ & $\checkmark$ & $\checkmark$ & $\checkmark$ & $x$ & & $x$ & $\checkmark$ \\
\hline Khan et al. [19] & pixel & X & $\checkmark$ & $\checkmark$ & $x$ & $\checkmark$ & $x$ & $x$ & $x$ & $\checkmark$ \\
\hline Shen et al. [31] & patch & $\checkmark$ & $\checkmark$ & $\checkmark$ & $x$ & & & $\checkmark$ & $\checkmark$ & $\checkmark$ \\
\hline Region based & level & SD & SS & HS & DS & DM & LD & SR & NR & $\mathbf{L}$ \\
\hline Guo et al. [16] & region & $\checkmark$ & $\checkmark$ & $\checkmark$ & $x$ & $\checkmark$ & $\checkmark$ & $\checkmark$ & $\checkmark$ & $\checkmark$ \\
\hline Vicente et al. [35] & region & & $x$ & $\checkmark$ & $\checkmark$ & & & $\checkmark$ & $x$ & $\checkmark$ \\
\hline Yuan et al. [40] & region & X & $\checkmark$ & $\checkmark$ & $\checkmark$ & $\checkmark$ & $\checkmark$ & $\checkmark$ & $x$ & $\checkmark$ \\
\hline Vicente et al. [36] & region & $\checkmark$ & $\checkmark$ & $\checkmark$ & $x$ & $\checkmark$ & & $\checkmark$ & $x$ & $\checkmark$ \\
\hline Sasi and Govindan [28] & region & $\checkmark$ & $\checkmark$ & $\checkmark$ & $x$ & & $x$ & $x$ & $\times$ & $\checkmark$ \\
\hline Color model based & level & SD & SS & HS & DS & DM & LD & SR & ER & $\mathbf{L}$ \\
\hline Salvador et al. [25] & edge & $\checkmark$ & & $\checkmark$ & $\checkmark$ & $\checkmark$ & $\checkmark$ & & $\checkmark$ & $x$ \\
\hline Figov et al. [6] & segment & X & $x$ & $\checkmark$ & $\checkmark$ & & $\checkmark$ & $\checkmark$ & $x$ & $x$ \\
\hline Xu et al. [39] & edge & $\checkmark$ & $\checkmark$ & $\checkmark$ & & & $x$ & & $x$ & $x$ \\
\hline Murali and Govindan [22] & pixel & $\checkmark$ & $\checkmark$ & $\checkmark$ & $\checkmark$ & $\checkmark$ & $x$ & & $x$ & $x$ \\
\hline Interactive based & level & SD & SS & HS & DS & DM & LD & SR & ER & $\mathbf{L}$ \\
\hline Wu and Tang [38] & pixel & & $\checkmark$ & $\checkmark$ & $\checkmark$ & $x$ & & & & $x$ \\
\hline Nielsen and Madsen [23] & pixel & & $\checkmark$ & $\checkmark$ & $\checkmark$ & $x$ & $\checkmark$ & & & $x$ \\
\hline Shor and Lischinski [32] & pixel & $\checkmark$ & $\checkmark$ & $\checkmark$ & $\checkmark$ & & $\checkmark$ & & & $x$ \\
\hline Arbel and Hel-Or [2] & pixel & $\checkmark$ & $\checkmark$ & $\checkmark$ & $\checkmark$ & & & & & $\checkmark$ \\
\hline Gong et al. [15] & region & & $\checkmark$ & $\checkmark$ & $\checkmark$ & & $x$ & & & $x$ \\
\hline
\end{tabular}

*SD-Self-shadow, SS-Detects Soft shadow, HS-Detects hard shadow, DS-Surface dependent, DM-Dark region misclassification, LD-Light/ camera dependent, IQ-Image quality, SR-Dependent on Segmentation results, NR-Non-adjacent regions, ER-Dependent on Edge detection results, L-Learning based, $\sqrt{ }$ - Yes, $X$ - No, M-moderate. 
Table 9

Shadow Detection - Qualitative results I: Row (i) has input images; (ii) and (iii) are detection results indicating success and failure cases respectively from the work mentioned under each image. Shadow regions/edges are represented as white in detection outputs (ii) and (iii)

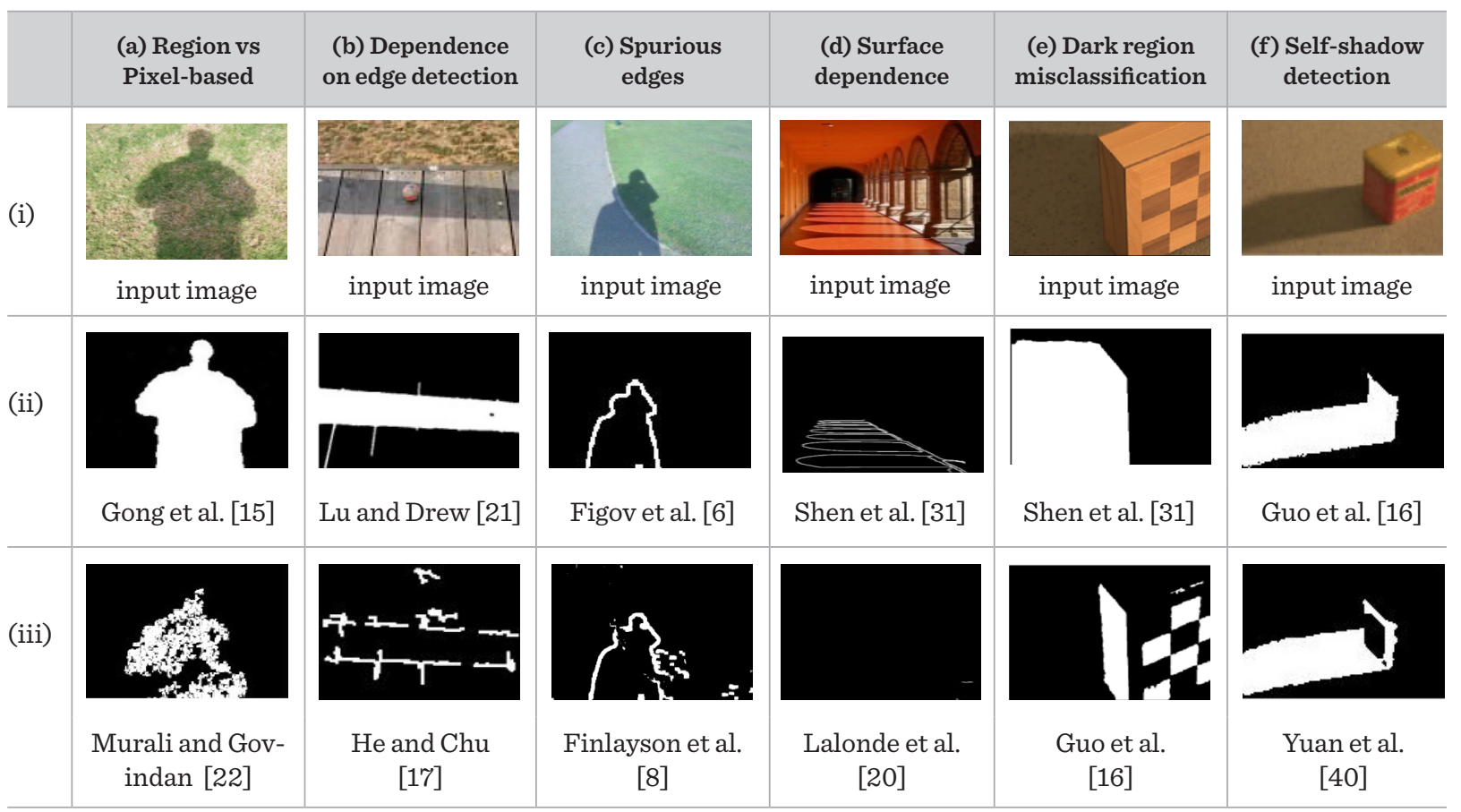

\section{Table 10}

Shadow Detection - Qualitative results II: Columns (a) and (c) are input images; (b) and (d) are detection results indicating success and failure cases respectively from the work mentioned under each image. Shadow region/edges are represented as white in (b) and (d)

\begin{tabular}{l|c|c|c|c|}
\hline (i) soft shadow & (b) \\
(ii) Complex scenes & input image & Golchin et al. [13] & Vicente et al. [36] \\
\hline input image
\end{tabular}

challenge in accurate detection of shadows [5].

Table 10(d)(i) illustrates the case in which extended soft shadows were undetected due to error in the initial segmentation since soft shadow boundaries are not detected by most of the segmentation or edge detection algorithms.

Table 10(ii) illustrates shadow detection in complex images. Table 10(b)(ii) gives a successful detection 
with very less misclassification. Table $10(\mathrm{~d})(\mathrm{ii})$ is a failure case due to overexposed regions.

\section{Quantitative Analysis}

Numerous metrics are available for evaluating the correctness of shadow detection results. Most of the authors use Per-pixel accuracy, Shadow Detection Rate, and Shadow Discrimination Rate. Matthews Correlation Coefficient (MCC) was used by Sasi and Govindan [28]. These metrics are computed using the number of True positives (TP), True negatives (TN), False positives (FP), and False negatives (FN). TP and TN indicate the number of correctly classified shadow pixels and non-shadow pixels, respectively. FP is the number of non-shadow pixels misclassified as shadow, and FN is the number of shadow pixels misclassified as non-shadow. The formula to compute each of the metrics is given below:

\begin{tabular}{l}
\hline Per - pixel Accuracy $=\frac{(T P+T N)}{N}$, \\
Shadow Detection Rate, $\eta=\frac{T P}{(T P+F N)}$ \\
Shadow Discrimination Rate, $\xi=\frac{T N}{(T N+F P)}$ \\
Mathews Correlation Coefficient, $M C C=$ \\
$=\frac{T P * T N-F P * F N}{(T P+F P)(T P+F N)(T N+F P)(T N+F N)^{\prime}}$
\end{tabular}

where, $N$ indicates the total number of pixels in the image.

The quantitative analysis based on three metrics, namely, Shadow Error Rate, Non-shadow Error Rate, and Balanced Error Rate are consolidated in Table 11. The Shadow Error Rate (SER) is computed from the number of misclassified shadow pixels. Non-shadow Error Rate(NER) is derived from the number of misclassified non-shadow pixels. Balanced Error Rate(BER) is computed as the mean of the Shadow Error Rate and Non-shadow Error Rate.

The formulae used to compute these values are given below:

Shadow Error Rate, $S E R=\frac{F N}{F N+T P}$,
Non-shadow Error Rate, $N E R=\frac{F P}{F P+T N}$,

(13)

Balanced Error Rate, $B E R=\frac{S E R+N E R}{2}$.

(14)

A lesser value for error rates indicates a better detection. The dataset for which evaluation is done is also included in the table.

Based on the results reported, it can be inferred from Table 11 that shadow detection by using Structured Convolutional Neural Network with linear optimization proposed by Shen et al. [31] outperforms all other techniques for the three datasets, namely, UIUC, UCF, and CMU. While the learning techniques deployed by feature based methods and region based methods provide good detection results, the time and memory consumption are higher for these methods.

Zhu et al. [41] mentioned that their method consumed approximately $9 \mathrm{~GB}$ for training 125 images of average size, $480 \times 320$. The automatic feature learning proposed by Khan et al. [19] requires 1GB memory and $\sim 1$ hour for training each of the above mentioned databases. The selection of the training images also contributes to the efficiency of detection algorithm.

\section{Conclusion}

Shadows are a natural phenomenon that appears on a surface due to obstruction of light by an object. Since their presence may cause complications in image vision applications, the detection and elimination of shadows have become an important topic of research. Shadow detection and removal is considered as an image enhancement technique and is therefore included in such pre-processing stage of computer vision applications as object detection and video examination. This makes it necessary to develop techniques that can detect and remove shadows accurately in very limited time.

Several works are available for shadow detection and removal from videos, multiple images, and single images. There also exist algorithms that are designed for a particular type of shadow. This includes algorithms for the detection of soft shadows, hard shadows, moving cast shadows, and shadows cast by a single object. Some of the existing works in shadow detection from a single image were reviewed in this paper. The tech- 
Table 11

A quantitative analysis of shadow detection methods

\begin{tabular}{|c|c|c|c|c|c|}
\hline Author & Detection Methods & Dataset & $\begin{array}{c}\text { Shadow } \\
\text { error rate }\end{array}$ & $\begin{array}{l}\text { Non-shadow } \\
\text { error rate }\end{array}$ & $\begin{array}{l}\text { Balanced } \\
\text { error rate }\end{array}$ \\
\hline Zhu et al. [41] & BCRF with BDT & $\mathrm{UCF}$ & 36.1 & 6.60 & 21.35 \\
\hline Panagopoulos et al. [24] & Bright Channel and MRF & $\mathrm{UCF}$ & 31.7 & 10.6 & 21.15 \\
\hline Lalonde et al. [20] & BDT, CRF and Scene Layout & CMU & 26.9 & 3.60 & 15.25 \\
\hline \multirow{4}{*}{ Guo et al. [16] } & \multirow{2}{*}{ Unary SVM } & $\mathrm{UCF}$ & 63.3 & 2.70 & 33.0 \\
\hline & & UIUC & 45.7 & 8.90 & 27.3 \\
\hline & \multirow{2}{*}{$\begin{array}{l}\text { Unary and } \\
\text { Pairwise SVM }\end{array}$} & $\mathrm{UCF}$ & 26.7 & 6.30 & 16.5 \\
\hline & & UIUC & 28.4 & 4.80 & 16.6 \\
\hline \multirow{4}{*}{ Vicente et al. [36] } & \multirow{2}{*}{ Leave-one-out optimization } & $\mathrm{UCF}$ & 22.9 & 6.20 & 14.5 \\
\hline & & UIUC & 14.9 & 4.20 & 9.50 \\
\hline & \multirow{2}{*}{ Leave-one-out optimization and MRF } & $\mathrm{UCF}$ & 20.0 & 6.40 & 13.2 \\
\hline & & UIUC & 9.90 & 4.40 & 7.20 \\
\hline \multirow{5}{*}{ Khan et al. [19] } & \multirow{2}{*}{ ConvNets } & $\mathrm{UCF}$ & 27.5 & 7.90 & 17.7 \\
\hline & & UIUC & 16.4 & 5.30 & 10.6 \\
\hline & \multirow{3}{*}{ ConvNets and CRF } & $\mathrm{UCF}$ & 22.0 & 7.40 & 14.7 \\
\hline & & $\mathrm{CMU}$ & 16.7 & 9.10 & 12.9 \\
\hline & & UIUC & 15.3 & 4.50 & 9.90 \\
\hline \multirow{3}{*}{ Shen et al. [31] } & \multirow{3}{*}{$\begin{array}{l}\text { Structured CNN with } \\
\text { Linear Optimization }\end{array}$} & $\mathrm{UCF}$ & 8.40 & 6.60 & 7.50 \\
\hline & & CMU & 8.40 & 2.30 & 5.35 \\
\hline & & UIUC & 8.70 & 4.97 & 6.83 \\
\hline
\end{tabular}

*The values were derived/taken from the papers discussed in this review

niques were classified under five categories, namely, invariant based detection, feature-based detection, region-based detection, color model based detection and interactive shadow detection. A qualitative and quantitative comparison is also included. The major observations made out of the survey may be stated as follows:

1 Even though the invariant-based shadow detection techniques provide results faster, the algorithms require prior knowledge of illuminant direction and high quality input images. Further, the results depend on the edge detection algorithm employed for generating edgemap.

2 Feature-based techniques provide better results with optimal feature set when compared to invariant based approach. However, the algorithms demand high computational and memory require- ments to train the classifier for detection.

3 Region-based shadow detection algorithms, although provide better results, demand higher computational requirements as the algorithms are based on learned features. Simple and fast color model based algorithms very often wrongly classify dark regions as shadows. Moreover, interactive shadow detection process, though appears to be simpler than automatic techniques, is not convenient for the user in case of multiple shadow regions. Soft shadows that span multiple surfaces also pose challenge in accurate detection of shadows.

4 Another major observation is that the accuracy of classifying a pixel (or region) as shadow is higher when the surrounding regions and their interac- 
tions are also considered. A single shadow might have different regions in it- the dark umbra region and the lighter penumbra region. An efficient detection algorithm must be capable of locating both

\section{References}

1. Al-Najdawi, N., Bez, H. E., Singhai, J., Edirisinghe, E. A. A Survey of Cast Shadow Detection Algorithms. Pattern Recognition Letters, 2012, 33(6), 752-764. http://doi. org/10.1016/j.patrec.2011.12.013.

2. Arbel, E., Hel-Or, H. Shadow Removal Using Intensity Surfaces and Texture Anchor Points. IEEE Transactions on Pattern Analysis and Machine Intelligence, 2011, 33(6), 1202-1216. https://doi.org/10.1109/TPAMI.2010.157

3. Barrow, H. G., Tenenbaum, J. M. Recovering Intrinsic Scene Characteristics from Images. Computer Vision Systems, 1978, 3-26.

4. Cucchiara, R., Grana, C., Piccardi, M., Prati, A. Detecting Moving Objects, Ghosts, and Shadows in Video Streams. IEEE Transactions on Pattern Analysis and Machine Intelligence, 2003, 25(10), 1337-1342. http:// doi.org/10.1109/TPAMI.2003.1233909.

5. Dong, Q., Liu, Y., Zhao, Q., Yang, H. Detecting Soft Shadows in a Single Outdoor Image: from Local Edge-Based Models to Global Constraints. Computers and Graphics, 2014, 38(1), 310-319. https://doi.org/10.1016/j. cag.2013.11.005

6. Figov, Z., Tal, Y., Koppel, M. Detecting and Removing Shadows. Proceedings of 7th IASTED International Conference on Computer Graphics and Imaging, 2004

7. Finlayson, G. D., Drew, M. S., Lu, C. Entropy Minimization for Shadow Removal. International Journal of Computer Vision, 2009, 85(1), 35-5\%. https://doi. org/10.1007/s11263-009-0243-z

8. Finlayson, G. D., Hordley, S. D. Color Constancy at a Pixel. Journal of the Optical Society of America, 2001, 18(2), 253-264. http://doi.org/10.1364/JOSAA .18.000253

9. Finlayson, G. D., Hordley, S. D., Drew, M. S. Removing Shadows from Images. Proceedings of the 7th European Conference on Computer Vision, 2002, 823-836. https://doi.org/10.1007/3-540-47979-1_55

10. Finlayson, G. D., Hordley, S. D., Lu, C., Drew, M. S. On the Removal of Shadows from Images. IEEE Transactions on Pattern Analysis and Machine Intelligence, 2006, the regions. Detection of self-shadows and heavily scattered shadows are also challenging. The risk of misclassifying dark objects as shadows should also be addressed.

11. Gevers, T., Stokman, H. Classifying Color Edges in Video into Shadow-Geometry, Highlight, or Material Transitions. IEEE Transactions on Multimedia, 2003, 5(2), 237-243. https://doi.org/10.1109/TMM.2003.811620

12. Gijsenij, A., Gevers, T. Shadow Edge Detection Using Geometric and Photometric Features. Proceedings of the 16th IEEE International Conference on Image Processing (ICIP), Cairo, Egypt, Nov 7-10, 2009, 693-696. http://doi.org/10.1109/ICIP.2009.5414089

13. Golchin, M., Khalid, F., Abdullah, L. N., Davarpanah, S. H. Shadow Detection Using Color and Edge Information. Journal of Computer Science, 2013, 9(11), 15751588. https://doi.org/10.3844/jcssp.2013.1575.1588

14. Gong, H., Cosker, D. P. Interactive Shadow Removal and Ground Truth for Variable Scene Categories. Proceedings of the British Machine Vision Conference, 2014. http://dx.doi.org/10.5244/C.28.36

15. Gong, H., Cosker, D., Li, C., Brown, M. User-Aided Single Image Shadow Removal. Proceedings of the IEEE International Conference on Multimedia and Expo(ICME), San Jose, CA, USA, July 15-19, 2013, 1-6. https:// doi.org/10.1109/ICME.2013.6607463

16. Guo, R., Dai, Q., Hoiem, D. Paired Regions for Shadow Detection and Removal. IEEE Transactions on Pattern Analysis and Machine Intelligence, 2013, 35(12), 29562967. http://doi.org/10.1109/TPA MI.2012.214

17. He, Q., Chu, C.-H. H. A New Shadow Removal Method for Color Images. Advances in Remote Sensing, 2013, r7-84. http://doi.org/10.4236/ars.2 013.22011

18. Jiang, X., Schofield, A., Wyatt, J. Shadow Detection Based on Colour Segmentation and Estimated Illumination. Proceedings of the British Machine Vision Conference, 2011, 1-11. http://doi.org/10.52 44/C.25.87

19. Khan, S. H., Bennamoun, M., Sohel, F., Togneri, R. Automatic Shadow Detection and Removal from a Single Image. IEEE Transactions on Pattern Analysis and Machine Intelligence, 2015, 38(3), 431-446. http://doi. 


\section{org/10.1109/TPAMI.2015.2462 355}

20. Lalonde, J. F., Efros, A. A., Narasimhan, S. G. Detecting Ground Shadows in Outdoor Consumer Photographs. In: Daniilidis K., et al. (Eds.), European Conference on Computer Vision, Lecture Notes in Computer Science, 6312. Springer, Berlin-Heidelberg, 322-335, 2010. http://d oi.org/10.1007/978-3-642-15552-9_24

21. Lu, C., Drew, M. S. Shadow Segmentation and Shadow-Free Chromaticity via Markov Random Fields. In: Color Imaging Conference, IS\&T - The Society for Imaging Science and Technology, 2005, 2005(1), 125-129.

22. Murali, S., Govindan, V. K. Shadow Detection and Removal from a Single Image Using LAB Color Space. Cybernetics and Information Technologies, 2013, 13(1), 95-103. https://doi.org/10.2478/cait-2013-0009

23. Nielsen, M., Madsen, C.B. Graph Cut Based Segmentation of Soft Shadows for Seamless Removal and Augmentation. In: Ersbøll, B.K., Pedersen, K. S. (Eds.), Scandinavian Conference on Image Analysis, Lecture Notes in Computer Science, 4522, Springer, Berlin-Heidelberg, 918-927, 2007. https://doi.org/10.1007/978-3540-73040-8_93

24. Panagopoulos, A., Wang, C., Samaras, D., Paragios, N. Estimating Shadows with the Bright Channel Cue. In: Kutulakos K. N. (Eds.) Trends and Topics in Computer Vision, ECCV 2010, Lecture Notes in Computer Science, 6554, Springer, Berlin-Heidelberg, 1-12, 2010. https://doi.org/10.10077/978-3-642-35740-4_1

25. Salvador, E., Cavallaro, A., Ebrahimi, T. Shadow Identification and Classification Using Invariant Color Models. Proceedings of IEEE International Conference on Acoustics, Speech, and Signal Processing, Salt Lake City, USA, 7-11 May, 2001, 3, 1545-1548. http://doi. org/10.1109/ICASSP.2001.941227

26. Salvador, E., Cavallaro, A., Ebrahimi, T. Cast Shadow Segmentation Using Invariant Color Features. Computer Vision and Image Understanding, 2004, 95(2), 238-259. http://doi.or g/10.1016/j.cviu.2004.03.008

27. Sanin, A., Sanderson, C., Lovell, B. C. Shadow detection: A Survey and Comparative Evaluation of Recent Methods. Pattern Recognition, 2012, 45(4), 1684-1695. http://doi.org/10.1016/j.patcog.2011.10.0 01

28. Sasi, R. K., Govindan, V. K. Fuzzy Split and Merge for Shadow Detection. Egyptian Informatics Journal, 2014, 16(1), 29-35. http://doi.org/10.1016/ j.eij.2014.11.003

29. Sasi, R. K., Govindan, V. K. Shadow Detection and Removal from Real Images: State of Art. Proceedings of the 3rd International Symposium on Women in Comput- ing and Informatics, Kochi, India, August 10 - 13, 2015 , 309-317. http://doi.org/10.1145/2791405.2791450

30. Serra, M., Penacchio, O., Benavente, R., Vanrell, M. Names and Shades of Color for Intrinsic Image Estimation. Proceedings of the IEEE Computer Society Conference on Computer Vision and Pattern Recognition, Providence, RI, USA, June 16-21, 2012, 278-285. http:// doi.org/10.1109/CVPR.2012.6247686

31. Shen, L., Chua, T. W., Leman, K. Shadow Optimization from Structured Deep Edge Detection. Proceedings of the IEEE Computer Society Conference on Computer Vision and Pattern Recognition, Boston, USA, June 07-12, 2015, 2067-2074. https://doi.org/10.1109/ CVPR.2015.7298818

32. Shor, Y., Lischinski, D. The Shadow Meets the Mask: Pyramid-Based Shadow Removal. Computer Graphics Forum, 2008, 27(2), 577-586. https://doi.org/10.1111/ j.1467-8659.2008.01155.x

33. Tian, J., Qi, X., Qu, L., Tang, Y. New Spectrum Ratio Properties and Features for Shadow Detection. Pattern Recognition, 2016, 51, 85-96. https://doi.org/10.1016/j. patcog.2015.09.006

34. Tian, J., Sun, J., Tang, Y. Tricolor Attenuation Model for Shadow Detection. IEEE Transactions on Image Processing, 2009, 18(10), 2355-2363. http://d oi.org/10.1109/TIP.2009.2026682

35. Vicente, T. F. Y., Yu, C.P., Samaras, D. Single Image Shadow Detection Using Multiple Cues in a Supermodular MRF. British Machine Vision Conference, 2013, 1-12. http://doi.org/10.524 4/C.27.126

36. Vicente, T. F. Y., Hoai, M., Samaras, D. Leave-One-Out Kernel Optimization for Shadow Detection. Proceedings of the IEEE International Conference on Computer Vision, Santiago, Chile, Dec 7-13, 2015, 3388-3396. http://doi.org/10.1109/ICCV.2015.387

37. Wu, Q., Zhang, W., Vijaya Kumar, B. V. K. Strong Shadow Removal via Patch-Based Shadow Edge Detection. Proceedings of IEEE International Conference on Robotics and Automation, Saint Paul, MN, USA, May 14-18, 2012, 2177-2182. https://doi.org/10.1109/ ICRA.2012.6224561

38. Wu, T. P., Tang, C. K. A Bayesian Approach for Shadow Extraction from a Single Image. Proceedings of the IEEE International Conference on Computer Vision, Beijing, China, Oct 17-21, 2005, 1, 480-487. http://doi. org/10.1109/ICCV.2 005.4

39. Xu, L., Qi, F., Jiang, R. Shadow Removal from a Single Image. Proceedings of 6th International Conference 
on Intelligent Systems Design and Applications (ISDA 2006), Jinan, China, Oct 6-18, 2006, 2, 1049-1054. http://doi.org/10.1109/ISDA .200 6.253756

40. Yuan, X., Ebner, M., Wang, Z. Single-Image Shadow Detection and Removal Using Local Colour Constancy Computation. IET Image Processing, 2015, 9(2), 118126. http://doi.org/10.1049/ietipr.2 014.0242
41. Zhu, J., Samuel, K. G. G., Masood, S. Z., Tappen, M. F. Learning to Recognize Shadows in Monochromatic Natural Images. Proceedings of the IEEE Conference on Computer Vision and Pattern Recognition (CVPR), San Francisco, CA, USA, June 13-18, 2010, 223-230. http://doi.org/10.1109/ CVPR.2010.5540209 